\title{
Role of van der Waals forces in the adsorption and diffusion of organic molecules on an insulating surface
}

\author{
O. H. Pakarinen, ${ }^{1,2}$ J. M. Mativetsky, ${ }^{3, *}$ A. Gulans, ${ }^{1}$ M. J. Puska, ${ }^{1}$ A. S. Foster, ${ }^{1,4}$ and P. Grutter ${ }^{3}$ \\ ${ }^{1}$ Department of Applied Physics, Helsinki University of Technology, P.O. Box 1100, FI-02015 TKK, Finland \\ ${ }^{2}$ Department of Physics and Helsinki Institute of Physics, University of Helsinki, P.O. Box 43, FI-00014 UH, Finland \\ ${ }^{3}$ Department of Physics, McGill University, Montreal, Canada H3A 2 T8 \\ ${ }^{4}$ Department of Physics, Tampere University of Technology, P.O. Box 692, FIN-33101 Tampere, Finland
}

(Received 28 April 2009; revised manuscript received 24 June 2009; published 3 August 2009)

\begin{abstract}
The adsorption and diffusion of 3,4,9,10-perylene-tetracarboxylic-dianhydride (PTCDA) molecules on a nanostructured $\mathrm{KBr}(001)$ surface were investigated by combining noncontact atomic force microscopy (NCAFM) and first-principles calculations. Atomically resolved measurements demonstrate trapping of PTCDA molecules in intentionally created rectangular monolayer-deep substrate pits and a preferential adsorption at kink sites. In order to understand the experimental results, we found that it was essential to include a firstprinciples treatment of the van der Waals interactions. We show that at some sites on the surface, $85 \%$ of the molecular binding is provided by van der Waals interactions, and in general it is always the dominant contribution to the adsorption energy. It also qualitatively changes molecular diffusion on the surface. Based on the specificity of the molecular interaction at kink sites, the species of the imaged ionic sublattice in the NC-AFM measurements could be identified.
\end{abstract}

DOI: 10.1103/PhysRevB.80.085401

PACS number(s): 68.37.Ps, 68.43.Fg, 68.43.Jk, 71.15.Mb

\section{INTRODUCTION}

Organic semiconductors are promising building blocks for organic electronic and optoelectronic devices. ${ }^{1}$ This is due to the ability of the molecules to form well-ordered crystalline films on various substrates by self-organization and to the possible control of the electronic properties by tuning the intramolecular structure and intermolecular assembly. ${ }^{2-4}$ Among candidate molecules, 3,4,9,10-perylenetetracarboxylic-dianhydride (PTCDA) is a prototype organic semiconductor that has been increasingly studied on insulating surfaces to provide the electrical isolation of the molecule from the substrate essential for device performance. ${ }^{5-11}$ In general, characterization of molecular ordering on the surface requires high-resolution imaging and is only possible on insulating surfaces with noncontact atomic force microscopy (NC-AFM). ${ }^{12}$ Earlier studies have shown that PTCDA islands with a well-defined herringbone arrangement form preferentially at the bottom of step edges. ${ }^{6}$ Moreover, atomic depth rectangular pits can be used as a means of trapping the molecules, resulting in islands which have the dimensions of the templating pits. ${ }^{7,8,13}$ To date, there is little known about the pit trapping mechanism, nor the nature of the bonding between organic molecules such as PTCDA and insulating surfaces in general-a topic that has proved controversial for PTCDA on metal surfaces. ${ }^{14-21}$

In order to investigate the nucleation and the trapping of PTCDA molecules, we used irradiation to controllably produce monolayer (ML) depth pits to act as trapping sites and used NC-AFM to study PTCDA in the submonolayer regime. Based on atomically resolved images, we show that the PTCDA preferentially adsorbs at kink sites. We use firstprinciples calculations of the adsorption and diffusion of PTCDA on KBr to explain the behavior of PTCDA on the surface, and we also show that it is critical to include an accurate model for the nonlocal correlations responsible for the strong van der Waals ( $\mathrm{vdW}$ ) interactions between the molecule and the surface. The role of van der Waals forces in surface diffusion has been explicitly demonstrated.

\section{METHODS}

\section{A. Experimental}

$\mathrm{KBr}(001)$ surfaces with monolayer deep rectangular pits were prepared by cleavage in ultrahigh vacuum (UHV), heating to $150{ }^{\circ} \mathrm{C}$ to remove residual surface charge and exposing the substrate to a calibrated dose of charge (1.3 $\left.\mu \mathrm{C} \mathrm{cm}^{-2}\right)$ to induce pit formation. ${ }^{22} \mathrm{~A}$ coverage of 0.4 ML of PTCDA was thermally evaporated onto the $\mathrm{KBr}$ surface at $300{ }^{\circ} \mathrm{C}$ after outgassing for $12 \mathrm{~h}$ at $220{ }^{\circ} \mathrm{C}$. $\mathrm{NC}$ AFM measurements were performed in the same UHV system at room temperature using a JEOL JSPM 4500a. In the NC-AFM mode, topography is measured by maintaining a constant shift in the cantilever oscillation frequency. ${ }^{12} \mathrm{~A}$ Nanosurf easyPLL was used to monitor the frequency shift and maintain a constant oscillation amplitude of $5 \mathrm{~nm}$. Silicon cantilevers (Nanosensors, PPP-NCLR) with a typical resonance frequency of $170 \mathrm{kHz}$, a spring constant of $40-50$ $\mathrm{nm}$, and a tip radius of under $10 \mathrm{~nm}$ were employed. In order to reduce the contribution of electrostatic forces during imaging, a voltage equal to the tip-sample contact potential difference was applied to the sample.

\section{B. Theoretical}

All calculations were carried out with the SIESTA code, ${ }^{23}$ which implements the density-functional theory (DFT) and the Perdew-Burke-Ernzerhof (PBE) exchange-correlation

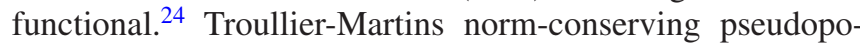
tentials were used for initial calculations. Conventional DFT approaches are intrinsically local and do not contain accurate descriptions of nonlocal correlation contributions. Since the latter is responsible for $\mathrm{vdW}$ interactions, known to be im- 
portant when studying the binding of conjugated carbon systems, ${ }^{25,26}$ we also performed calculations using the selfconsistent implementation of the first-principles vdW density functional (vdW-DF) with an adaptive real-space grid approach $^{27}$ employing nonlocal correlations. ${ }^{28}$ Previous studies have shown that the magnitude of $\mathrm{vdW}$ interaction is somewhat dependent on the choice of exchange functional used $^{27,29}$ and it was shown that in systems where the bonding is dominated by the $\mathrm{vdW}$ interaction, the choice of the revPBE (Ref. 30) exchange functional is favorable, while for hydrogen bonded systems the PBE exchange functional provided better accuracy. The bonding of PTCDA to $\mathrm{KBr}$ involves both the vdW and the electrostatic interactions between ions; therefore, we performed all the vdW-DF calculations with both approximations for the exchange and found that it led to a constant difference of about $0.5 \mathrm{eV}$ in adsorption energies, but the choice does not affect our conclusions. We also used the original PBE relaxed geometries for the vdW-DF calculations, but the additional relaxations introduced by the vdW-DF lead to changes in the adsorption energy of less than $0.1 \mathrm{eV}$.

To aid in computational efficiency, we defined two levels of accuracy within the calculations- "high" accuracy for the most important points of the diffusion path, all the adsorption energy calculations and as a general benchmark, and "low" accuracy for the majority of the points on the diffusion path. At high accuracy, we used a double $\zeta$ with polarization basis set for all orbitals and a $3 \times 3 \times 1 k$-point grid. For low accuracy, the polarization orbital was removed for $\mathrm{C}, \mathrm{H}$, and $\mathrm{Br}$, and we included only the gamma point-recalculation of key points showed that this did not significantly affect the relaxed structure. Extensive testing demonstrated that a mesh cutoff of $175 \mathrm{Ry}$ and an energy shift of $25 \mathrm{meV}$ provided good general accuracy and were used in all calculations. This energy shift value ensures a large spatial extent of the basis orbitals, which is important for preventing severe overestimation of binding. ${ }^{14,15}$ For all configurations considered in high accuracy, we also calculated the vdW contribution to the adsorption energy and corrected for any basis set superposition error (BSSE). For the remaining points on the diffusion path, we interpolated from the conventional DFT result according to the most relevant high-accuracy point-the coincidence of the final high and low accuracy points along the diffusion path supports the validity of this approximation.

We used $3 \times 4$ or $3 \times 6$ unit cells of $\mathrm{KBr}$ to define the surface, the step or the kink systems, and these were found to be large enough to minimize the interaction of neighboring periodic images of the molecule. A surface depth of three atomic layers with the lowest layer fixed was sufficient to allow the top layers to relax naturally in simulations. The forces on atoms were relaxed to within a tolerance of $0.02 \mathrm{eV} / \AA$. Diffusion energetics were studied with the drag method, ${ }^{31}$ where we calculate the total energy of the system as a function of stepwise movement of the molecule in steps of one tenth of the lattice spacing $(0.35 \AA)$, at each point relaxing the unconstrained forces of the molecule and the surface below $0.02 \mathrm{eV} / \AA$, while we constrain the relaxation of two neighboring atoms at the center of the molecule to a plane perpendicular to the direction of this movement.
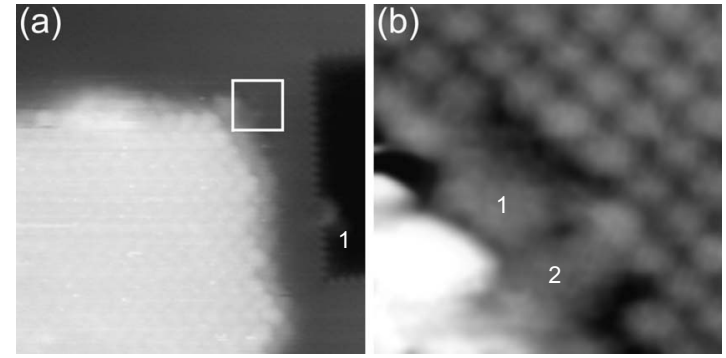

FIG. 1. High-resolution NC-AFM measurements showing (a) a three-layer PTCDA island inside a monolayer-deep rectangular pit and part of a nearly empty pit with one or two molecules at a kink site (labeled 1) $\left(20 \times 20 \mathrm{~nm}^{2}\right)$, (b) zoom in to area shown by box in (a), revealing two first layer PTCDA molecules (labeled 1 and 2) at the edge of an island adsorbed at kink sites $\left(3 \times 3 \mathrm{~nm}^{2}\right)$.

\section{RESULTS}

As a first step, we considered in detail the possible adsorption sites of PTCDA on the surface. In experimental AFM images, we observe that PTCDA molecules preferentially trap in the pits of irradiated alkali halide surfaces forming regularly arrayed islands, either completely or partially filling pits, or even overfilling them. ${ }^{8}$ Islands are not formed on the atomically flat terraces of the surface, and isolated molecules are never observed on the terraces or at straight step edges. However, we have occasionally observed them at the edge of pits at kink sites. Figure 1(a) shows an example of a rectangular trapped island, with an adjacent pit which is mostly empty, but contains what appears to be one or two molecules at a kink site. Moreover, although most of the PTCDA island is three layers high, some first and second layer molecules are also resolved along the edges. Tip doubling effects can be ruled out since there are features at the upper layer edges which are not repeated in the other layers. In addition, the size and the height of the molecules in each layer correspond to the expected dimensions for PTCDA. A high-resolution image of two first layer molecules along with the adjacent $\mathrm{KBr}$ ions is shown in Fig. 1(b). Since we know that NC-AFM images of cubic ionic surfaces will always show only one ionic sublattice as bright, ${ }^{32}$ we cannot easily identify the ions seen at this point, but we can clearly see that the step edge in this region is formed from two equivalent kinks, demonstrating the increased contrast characteristic of low-coordinated sites. ${ }^{33}$ Below the step edge, we observe two PTCDA molecules in registry with the kink sites - this is a strong indication that the kink sites within pits act as the preferred adsorption sites. To explore this further, we calculated the adsorption energy of PTCDA in about 20 different configurations on the terrace, at the step edge and at the kinks on the surface.

Figure 2 shows six of the configurations we considered in the calculations. Noting that no covalent bonds are formed between PTCDA and the surface, the key components of the interaction are the ionic attraction between the terminating $\mathrm{O}$ atoms and $\mathrm{K}^{+}$ions in the surface and the vdW attraction between the molecule and the surface. In Figs. 2(a)-2(c), we compare three different orientations of PTCDA on a $\mathrm{KBr}$ terrace, where the balance between these two factors can be 


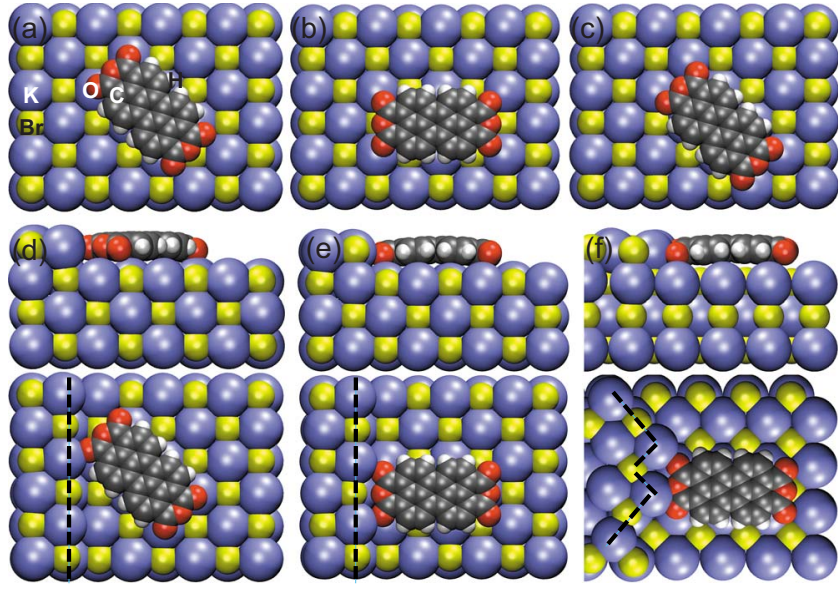

FIG. 2. (Color online) Calculated adsorption sites of PTCDA on the $\mathrm{KBr}(001)$ surface in different orientations (a)-(c) on a terrace, $[(\mathrm{d}),(\mathrm{e})]$ at a step edge, and (f) at a kink. The dashed lines label the edge of the upper terrace.

clearly seen. On the terrace, the vdW component of the interaction depends mainly on the surface area covered by the molecule and this is almost independent of orientation, as the large cost of changing the $\mathrm{C}-\mathrm{C}$ bond length means the molecule's size remains consistent at all sites. However, orientation strongly affects the matching between $\mathrm{O}$ atoms and surface $\mathrm{K}^{+}$ions. In Figs. 2(a) and 2(b) the configuration forces at least one of the $\mathrm{O}$ atoms into close proximity to a $\mathrm{Br}^{-}$ion in the surface, whereas in Fig. 2(c) the oxygen atoms match well with surface $\mathrm{K}^{+}$ions and this has a larger adsorption energy (see Table I). Similar factors control adsorption at the step edge, but the low coordination of atoms at the step edge increases the $\mathrm{O}-\mathrm{K}^{+}$interaction at step sites, so that the molecule is constrained to fewer possible configurations. Figures 2(d) and 2(e) show the two stable step-edge configurations

TABLE I. Calculated contributions to the molecular adsorption energy in various configurations: PBE is the adsorption energy for the PBE functional, vdW is the additional contribution from vdW$\mathrm{DF}$, and $E_{A}$ (total) is the total adsorption energy (in eV). All values are BSSE corrected. "Sites" refer to Figs. 2 and 3.

\begin{tabular}{cccc}
\hline \hline Site & PBE & vdW & $E_{A}$ (total) \\
\hline $2(\mathrm{a})$ & -0.29 & -1.61 & -1.90 \\
$2(\mathrm{~b}) / 3(\mathrm{j})$ & -0.19 & -1.63 & -1.82 \\
$2(\mathrm{c}) / 3(\mathrm{a})$ & -0.51 & -1.86 & -2.36 \\
$2(\mathrm{~d}) / 3(\mathrm{~h})$ & -0.31 & -1.80 & -2.11 \\
$2(\mathrm{e})$ & -0.33 & -1.85 & -2.18 \\
$2(\mathrm{f})$ & -0.96 & -1.89 & -2.85 \\
$3(\mathrm{~b})$ & -0.55 & -1.66 & -2.21 \\
$3(\mathrm{c})$ & -0.31 & -1.29 & -1.60 \\
$3(\mathrm{~d})$ & -0.24 & -1.39 & -1.63 \\
$3(\mathrm{e})$ & -0.52 & -1.47 & -1.99 \\
$3(\mathrm{f})$ & -0.45 & -1.43 & -1.88 \\
$3(\mathrm{~g})$ & -0.55 & -1.17 & -1.72 \\
$3(\mathrm{i})$ & -0.19 & -0.32 & -0.51 \\
\hline \hline
\end{tabular}

we found in our calculations. In both cases, at least one $\mathrm{O}$ atom is in close proximity to $\mathrm{a} \mathrm{Br}^{-}$ion in the surface, and it is energetically more favorable to adsorb on the terrace (see Table I). The only case where the strong interaction between low-coordinated surface $\mathrm{K}^{+}$ions and $\mathrm{O}$ atoms compensates for any mismatch is at a kink site [see Fig. 2(f)], where one end of the molecule bonds with two low-coordinated $\mathrm{K}^{+}$ ions, resulting in the largest adsorption energy of all the configurations. This result also makes it possible to identify the ions in the NC-AFM measurements. In Fig. 1(b), the PTCDA molecules adsorbed at $\mathrm{KBr}$ kink sites are aligned with the corrugation maxima (bright spots) of the imaged substrate lattice. In view of the strongly favored interaction between the $\mathrm{K}^{+}$kink ions and the $\mathrm{O}$ atoms at the ends of the PTCDA, the bright spots must be the $\mathrm{K}^{+}$ions. The specificity of the PTCDA-KBr interaction at kinks thus represents a means of using PTCDA as a marker for removing ambiguity about the species of the imaged sublattice.

Table I shows clearly the importance of the vdW contribution to the total adsorption energy, providing $85 \%$ of the interaction for sites 2(b), 2(d), and 3(d). In fact, without the vdW contribution, at many sites on the surface, it is likely that the molecule would easily desorb at room temperature. As one would expect, the vdW interaction is maximized when the molecule is flat to the surface, e.g., 2(e) and 2(f), while it is significantly reduced when a significant part of the molecule is far from the surface, e.g., 3(g) and 3(i).

Although the study of adsorption already provides some links to the observation of single molecules at kink sites in pits, to understand the formation and the nucleation of islands of PTCDA molecules, we also need to look at its diffusion. The fact that we only observe molecules at kinks or in islands, and never isolated on the terraces or at the straight step edges, implies that the molecules are highly mobile after adsorption. On the terraces, starting from the configuration shown in Fig. 2(c), our calculations predict a barrier for molecular migration of about $0.4 \mathrm{eV}$ and PTCDA will certainly be mobile at room temperature on the time scale of an $\mathrm{NC}$ AFM image. This means that deposited molecules will migrate across the surface and then, depending on whether they are within a pit or not, will encounter a step edge from below or above, respectively. Figure 3 shows the calculated diffusion barriers for a PTCDA molecule in different orientations encountering a step edge-note that at finite temperature the molecule would adopt the configuration of highest adsorption energy ("most negative") at each point and "cross" between the curves. As on the surface, the energetics is strongly dependent on the registry of the molecule with the $\mathrm{KBr}$ lattice. Initially the molecule is in the [110] orientation with all $\mathrm{O}$ atoms in registry with surface $\mathrm{K}^{+}$ions [see Fig. 3(a)] and this configuration remains favorable as the molecule begins crossing the step edge. Adsorption above the step edge is less favorable than adsorption on the terrace, although the difference is only about $0.1 \mathrm{eV}$ (see Table I). At the step edge itself, there is a barrier of about $0.4 \mathrm{eV}$ for the molecule to cross the plane of the step, and it is stable in this position [see Fig. 3(b)] at about $0.2 \mathrm{eV}$ lower adsorption energy than on the terrace. As the molecule is pushed across the step edge the energy magnitude reduces steadily, with local minima as the $\mathrm{O}$ atoms move in and out of registry with the surface $\mathrm{K}^{+}$ 


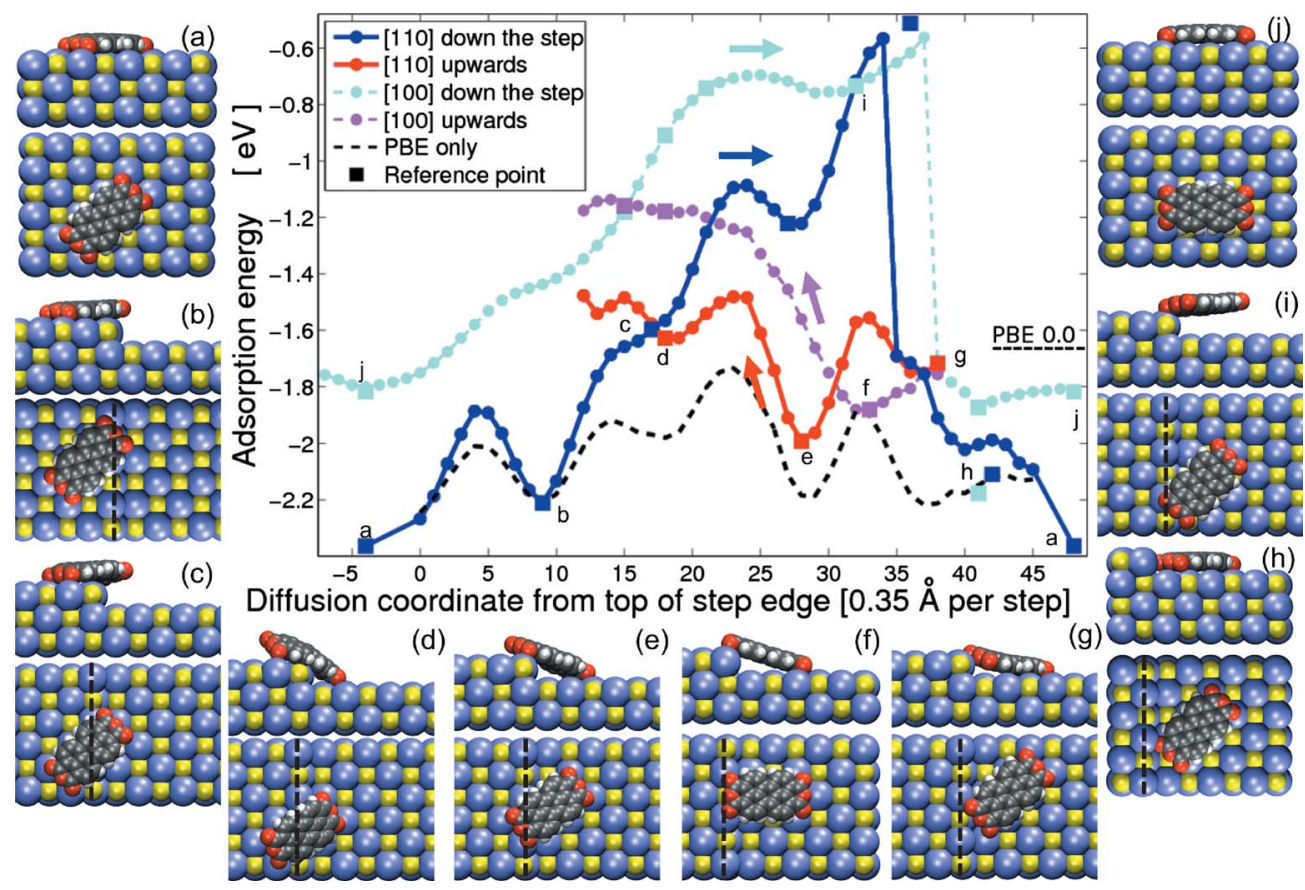

FIG. 3. (Color online) Calculated diffusion barriers plotted in terms of adsorption energy and corresponding simulation snapshots for a PTCDA molecule to cross a step edge on the $\mathrm{KBr}(001)$ surface. The curves are calculated in two orientations of the molecule, referred to as [110] and [100], according to the axis of the molecule. The blue (dark continuous) and cyan (light dashed) curves are for the molecule diffusing down the step, while red (gray continuous) and magenta (gray dashed) are for upward diffusion. Reference points labeled in the plot are calculated at high accuracy. The PBE curve includes BSSE corrections and has been shifted downward by $1.66 \mathrm{eV}$ for clarity.

ions. At the position shown in Fig. 3(c), the molecule reaches the tilt point, where the end of the molecule should now drop to the lower terrace in the configuration shown in Fig. 3(d). However, due to the absence of thermal fluctuations to break the system from the local minimum, it will actually remain horizontal until it is only bound by a single $\mathrm{O}$ to the step edge [see Fig. 3(i)]. This is clearly unphysical, and diffusion simulations in the reverse direction, up the step edge, show that the molecule prefers to tilt down to the surface. As the molecule migrates further across the step edge, we predict that it actually undergoes an orientation change from [110] in Fig. 3(e) to [100] in Fig. 3(f) in an effort to increase O-K ionic bonding. The two orientations are very close in energy for the next few points [see Fig. 3(g)], but the [110] orientation is again favorable as the molecule moves closer to the equilibrium terrace position [see Fig. 3(h)]. The maximum barrier to cross the step from the upper to the lower terrace is about $0.6 \mathrm{eV}$ [Figs. 3(b)-3(d)], while the reverse process has a similar maximum barrier of about $0.6 \mathrm{eV}$ [right hand, Figs. 3(a)-3(f)]. Interestingly, in the "PBE only" curve, the highest barrier $(>0.4 \mathrm{eV})$ becomes 3(a) to 3(b), as the absence of $\mathrm{vdW}$ forces means it is easier to cross the step- $-\mathrm{vdW}$ interactions have introduced a qualitative change in the diffusion barriers.

Since there is no barrier height asymmetry at the step edge to cause pit trapping, a more likely mechanism involves molecular diffusion over the surface and across steps until a kink site is reached. The kink site is about $0.5 \mathrm{eV}$ higher in adsorption energy than the terrace and $0.7 \mathrm{eV}$ higher than the step edge. Thus, it is likely that pit trapping proceeds via nucleation of the molecules at kink sites, which then act as anchors for subsequent island growth, bounded by the pit edges. The existence of a PTCDA molecule at a kink may also provide a new channel for diffusion down the step, since PTCDA molecules are highly mobile on top of a PTDCA film. ${ }^{34}$ The observation that many surface pits remain empty, despite the fact that the PTCDA lands evenly over the surface during deposition, is consistent with the relatively small calculated diffusion barrier for crossing steps.

\section{CONCLUSIONS}

Our results demonstrate that PTCDA molecules at low density will adsorb strongly at the kink sites within pits on the $\mathrm{KBr}(001)$ surface and are generally mobile at other sites on the surface. Interestingly, this also provides direct interpretation of the high-resolution NC-AFM images, as the kink sites must be cation kinks and, hence, in this case $\mathrm{K}^{+}$ions are shown as bright contrast in images. Although previous studies have used marker molecules ${ }^{35,36}$ to interpret NC-AFM contrast, here it has been achieved in a single experiment at room temperature. We also show that it is essential to include an accurate treatment of the van der Waals interaction to understand the adsorption and diffusion of the molecule.

\section{ACKNOWLEDGMENTS}

We acknowledge the support from the Academy of Finland, the ESF FANAS program, and CSC (O.H.P., A.G., M.J.P., and A.S.F.) and NSERC, CIfAR, FQRNT, and CFI (J.M.M. and P.G.). We thank S. A. Burke and S. Fostner for helpful discussions. 
*Present address: Institut de Science et d'Ingénierie Supramoléculaires (ISIS), CNRS 7006, Université de Strasbourg, 8 allée Gaspard Monge, 67000 Strasbourg, France.

${ }^{1}$ C. Dimitrakopoulos and P. Malenfant, Adv. Mater. 14, 99 (2002).

${ }^{2}$ N. Karl, Synth. Met. 133-134, 649 (2003).

${ }^{3}$ G. Witte and C. Wöll, J. Mater. Res. 19, 1889 (2004).

${ }^{4}$ S. A. Burke, J. M. LeDue, J. M. Topple, S. Fostner, and P. Grütter, Adv. Mater. 21, 2029 (2009).

${ }^{5}$ D. Schlettwein, A. Back, B. Schilling, and T. Fritz, Chem. Mater. 10, 601 (1998).

${ }^{6}$ T. Kunstmann, A. Schlarb, M. Fendrich, T. Wagner, R. Möller, and R. Hoffmann, Phys. Rev. B 71, 121403(R) (2005).

${ }^{7}$ J. M. Mativetsky, S. A. Burke, S. Fostner, and P. Grütter, Small 3, 818 (2007).

8 J. M. Mativetsky, S. A. Burke, S. Fostner, and P. Grutter, Nanotechnology 18, 105303 (2007).

${ }^{9}$ T. Roll, T. Kunstmann, M. Fendrich, R. Moeller, and M. Schleberger, Nanotechnology 19, 045703 (2008).

${ }^{10}$ T. Dienel, C. Loppacher, S. C. B. Mannsfeld, R. Forker, and T. Fritz, Adv. Mater. 20, 959 (2008).

${ }^{11}$ S. A. Burke, W. Ji, J. M. Mativetsky, J. M. Topple, S. Fostner, H. J. Gao, H. Guo, and P. Grutter, Phys. Rev. Lett. 100, 186104 (2008).

${ }^{12}$ F. J. Giessibl, Rev. Mod. Phys. 75, 949 (2003).

${ }^{13}$ L. Nony, R. Bennewitz, O. Pfeiffer, E. Gnecco, A. Baratoff, E. Meyer, T. Eguchi, A. Gourdon, and C. Joachim, Nanotechnology 15, S91 (2004).

${ }^{14}$ R. Rurali, N. Lorente, and P. Ordejon, Phys. Rev. Lett. 95, 209601 (2005).

${ }^{15}$ A. Hauschild, K. Karki, B. C. C. Cowie, M. Rohlfing, F. S. Tautz, and M. Sokolowski, Phys. Rev. Lett. 95, 209602 (2005).

${ }^{16}$ S. X. Du, H. J. Gao, C. Seidel, L. Tsetseris, W. Ji, H. Kopf, L. F. Chi, H. Fuchs, S. J. Pennycook, and S. T. Pantelides, Phys. Rev. Lett. 97, 156105 (2006).

${ }^{17}$ A. Kraft, R. Temirov, S. K. M. Henze, S. Soubatch, M. Rohlfing, and F. S. Tautz, Phys. Rev. B 74, 041402(R) (2006).

${ }^{18}$ M. Rohlfing, R. Temirov, and F. S. Tautz, Phys. Rev. B 76, 115421 (2007).

${ }^{19}$ P. Sony, P. Puschnig, D. Nabok, and C. Ambrosch-Draxl, Phys.
Rev. Lett. 99, 176401 (2007).

${ }^{20}$ M. Rohlfing and T. Bredow, Phys. Rev. Lett. 101, 266106 (2008).

${ }^{21}$ L. Romaner, D. Nabok, P. Puschnig, E. Zojer, and C. AmbroschDraxl, New J. Phys. 11, 053010 (2009).

${ }^{22}$ J. M. Mativetsky, Yoichi Miyahara, Shawn Fostner, Sarah A. Burke, and Peter Grutter, Appl. Phys. Lett. 88, 233121 (2006).

${ }^{23}$ J. M. Soler, Emilio Artacho, Julian D. Gale, Alberto García, Javier Junquera, Pablo Ordejón, and Daniel Sánchez-Portal, J. Phys.: Condens. Matter 14, 2745 (2002).

${ }^{24}$ J. P. Perdew, K. Burke, and M. Ernzerhof, Phys. Rev. Lett. 77, 3865 (1996).

${ }^{25}$ H. Rydberg, M. Dion, N. Jacobson, E. Schroder, P. Hyldgaard, S. I. Simak, D. C. Langreth, and B. I. Lundqvist, Phys. Rev. Lett. 91, 126402 (2003).

${ }^{26}$ K. Johnston, J. Kleis, B. I. Lundqvist, and R. M. Nieminen, Phys. Rev. B 77, 121404(R) (2008).

${ }^{27}$ A. Gulans, M. J. Puska, and R. M. Nieminen, Phys. Rev. B 79, 201105(R) (2009).

${ }^{28}$ M. Dion, H. Rydberg, E. Schröder, D. C. Langreth, and B. I. Lundqvist, Phys. Rev. Lett. 92, 246401 (2004).

${ }^{29}$ O. A. Vydrov, Q. Wu, and T. V. Voorhis, J. Chem. Phys. 129, 014106 (2008).

${ }^{30}$ Y. Zhang and W. Yang, Phys. Rev. Lett. 80, 890 (1998).

${ }^{31}$ H. Jónsson, G. Mills, and K. W. Jacobsen, Classical and Quantum Dynamics in Condensed Phase Simulations (World Scientific, Singapore, 1998).

${ }^{32}$ W. Hofer, A. S. Foster, and A. L. Shluger, Rev. Mod. Phys. 75, 1287 (2003).

${ }^{33}$ R. Bennewitz, A. S. Foster, L. N. Kantorovich, M. Bammerlin, C. Loppacher, S. Schar, M. Guggisberg, E. Meyer, and A. L. Shluger, Phys. Rev. B 62, 2074 (2000).

${ }^{34}$ S. Yim, K. il Kim, and T. S. Jones, J. Phys. Chem. C 111, 10993 (2007).

${ }^{35}$ K. I. Fukui, H. Onishi, and Y. Iwasawa, Chem. Phys. Lett. 280, 296 (1997).

${ }^{36}$ J. V. Lauritsen, Adam S. Foster, Georg H. Olesen, Mona C. Christensen, Angelika Kühnle, Stig Helveg, Jens R. RostrupNielsen, Bjerne S. Clausen, Michael Reichling, and Flemming Besenbacher, Nanotechnology 17, 3436 (2006). 